$5-1-2014$

\title{
Likelihood Ratio Type Test for Linear Failure Rate Distribution vs. Exponential Distribution
}

\author{
R R. L. Kantam \\ Acharya Nagarjuna University, India, kantam.rrl@gmail.com \\ M C. Priya \\ Acharya Nagarjuna University, India \\ M S. Ravikumar \\ Acharya Nagarjuna University, India, msrk.raama@gmail.com
}

Follow this and additional works at: http://digitalcommons.wayne.edu/jmasm

Part of the Applied Statistics Commons, Social and Behavioral Sciences Commons, and the Statistical Theory Commons

\section{Recommended Citation}

Kantam, R R. L.; Priya, M C.; and Ravikumar, M S. (2014) "Likelihood Ratio Type Test for Linear Failure Rate Distribution vs. Exponential Distribution," Journal of Modern Applied Statistical Methods: Vol. 13 : Iss. 1 , Article 11.

DOI: $10.22237 /$ jmasm/1398917400

Available at: http://digitalcommons.wayne.edu/jmasm/vol13/iss1/11

This Regular Article is brought to you for free and open access by the Open Access Journals at DigitalCommons@WayneState. It has been accepted for inclusion in Journal of Modern Applied Statistical Methods by an authorized editor of DigitalCommons@WayneState. 


\section{Likelihood Ratio Type Test for Linear Failure Rate Distribution vs. Exponential Distribution}

\author{
R. R. L. Kantam \\ Acharya Nagarjuna University \\ Guntur-Andhra Pradesh, India
}

\author{
M. C. Priya \\ Acharya Nagarjuna University \\ Guntur-Andhra Pradesh, India
}

\author{
M. S. Ravikumar \\ Acharya Nagarjuna University \\ Guntur-Andhra Pradesh, India
}

The Linear Failure Rate Distribution (LFRD) is considered. The graphs of its probability density function are examined for selected parameter combinations. Some of them are similar to the well-known exponential distribution. Incidentally exponential distribution is one of the two component models of the LFRD model. In view of the simpler form of exponential model as applicable in inference, looking at the frequency curves of LFRD, a test statistic is proposed based on ratio of likelihood functions containing the standard forms of the density functions of both LFRD and Exponential to discriminate between LFRD and exponential models. The critical values and the powers of the test statistic are developed.

Keywords: $\quad$ Linear failure rate distribution, likelihood ratio type, test statistic, power

\section{Introduction}

In reliability studies, series systems are one of many popular system configurations. If a series system has two components having independently distributed lifetime random variables with failure rate functions $h_{1}(x)$ and $h_{2}(x)$ then the reliability of the series system is

$$
R(x)=\exp \left[-\int_{0}^{x}\left\{h_{1}(t)+h_{2}(t)\right\} d t\right]
$$

The corresponding cumulative distribution function, failure density function and failure rate function are respectively given by

Dr. Kantam is a Professor in the Department of Statistics. Email him at kantam.rrl@gmail.com.M. S. Ravikumar is a UGC Research Fellow in the Department of Statistics. Email him at: msrk.raama@gmail.com. 


$$
\begin{gathered}
F(x)=1-\exp \left[-\int_{0}^{x}\left\{h_{1}(t)+h_{2}(t)\right\} d t\right] \\
f(x)=\frac{d}{d x} F(x) \\
h(x)=\frac{f(x)}{R(x)}
\end{gathered}
$$

Taking $h_{1}(x), h_{2}(x)$, as the failure rates of the exponential and Rayleigh distributions in (1) results in the most commonly used Linear Failure Rate Distribution (LFRD). More specifically, if $h_{1}(x)=a$ and $h_{2}(x)=b x$ then the failure density function, cumulative distribution function, hazard or failure rate function of LFRD is:

$$
\begin{gathered}
f(x)=(a+b x) e^{-\left(a x+\frac{b x^{2}}{2}\right)} ; x>0, a>0, b>0 \\
F(x)=1-e^{-\left(a x+\frac{b x^{2}}{2}\right)} ; x>0, a>0, b>0 \\
h(x)=a+b x
\end{gathered}
$$

Bain (1974) seems to be one of the earliest works that has touched upon LFRD as a model useful for analysis in life testing. Ananda Sen (2005) gave a detailed review along with the distributional characteristics and inferential aspects of LFRD. Some basic features of LFRD are as follows:

Mean:

$$
\mu=\sqrt{\frac{2 \pi}{b} e^{\frac{a^{2}}{2 b}}\left(1-\phi\left(\frac{a}{\sqrt{b}}\right)\right.}
$$

where $\phi$ denotes the cumulative distribution function of a standard normal variate. 
Variance:

$$
\sigma^{2}=\frac{2}{b}(1-a \mu)-\mu^{2}
$$

Mode:

$$
M=\left(\sqrt{\frac{1}{b}-\frac{a}{b}}\right) I\left(a^{2}<b\right)
$$

where $I($.$) denotes indicator function.$

$100 \mathrm{p}^{\text {th }}$ Percentile:

$$
F^{-1}(p)=\sqrt{\left(\frac{a}{b}\right)^{2}-\frac{2 \log (1-p)}{b}}-\frac{a}{b}
$$

and hence median is

$$
M_{d}=\sqrt{\left(\frac{a}{b}\right)^{2}-\frac{2 \log (0.5)}{b}}-\frac{a}{b}
$$

In biological sciences this is called $50 \%$ survival time denoted by $t_{50}$.

Recurrence relation for raw moments is

$$
\mu_{k}^{1}=\frac{a}{k+1} \mu_{k+1}^{\prime}+\frac{b}{k+2} \mu_{k+2}^{\prime} ; \quad k=0,1,2 \ldots
$$

The second, third and fourth non-central moments are

$$
\begin{gathered}
\mu_{2}^{\prime}=\frac{2}{b}(1-a \mu) \\
\mu_{3}^{\prime}=\frac{3}{b}\left(\mu-\frac{a}{b}(1-a \mu)\right)
\end{gathered}
$$




$$
\mu_{4}^{\prime}=\frac{8}{b^{2}}+\frac{4 a^{2}}{b^{3}}-\mu\left(\frac{12 a}{b^{2}}+\frac{4 a^{3}}{b^{3}}\right)
$$

where $\mu$ is the mean of the distribution given by (8).

It can be seen from (10) that LFRD has a non-zero mode only if its parameters $a$ and $b$ satisfy the relation $a^{2}<b$ with $a>0, b>0$.

The graphs of LFRD density function for some combinations of the parameters $a, b$ are shown in the following figures.

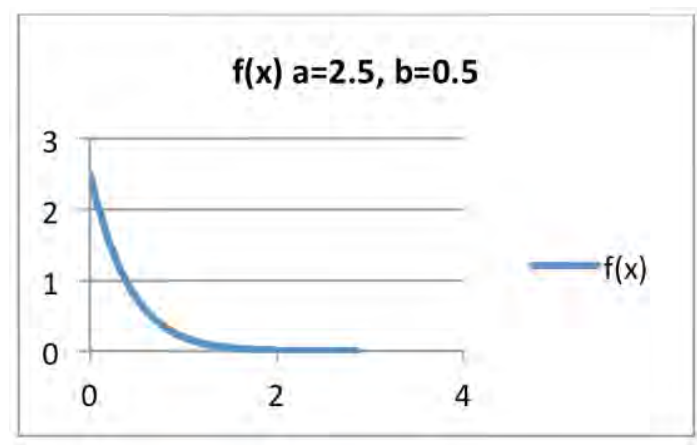

Figure 1. LFRD Density function when $a=2.5, b=0.5$

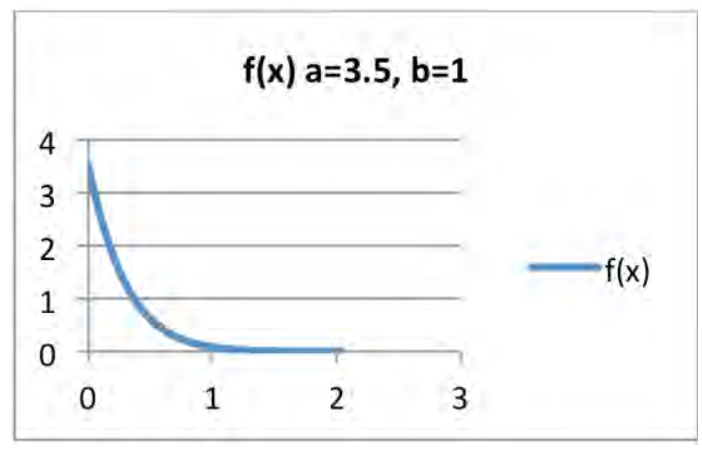

Figure 3. LFRD Density function when $a=3.5, b=1$

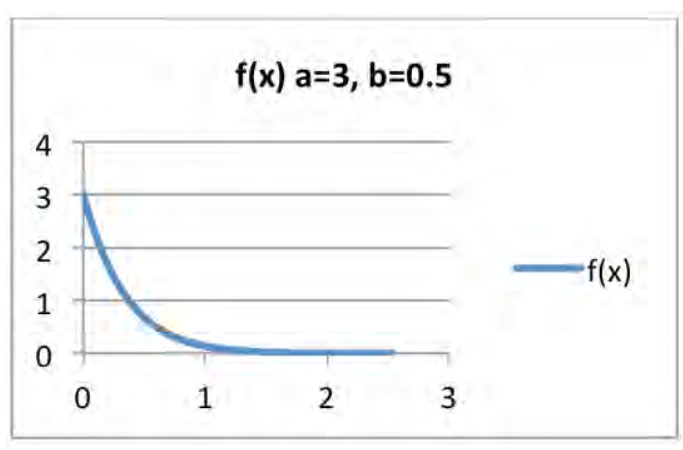

Figure 2. LFRD Density function when $a=3, b=0.5$

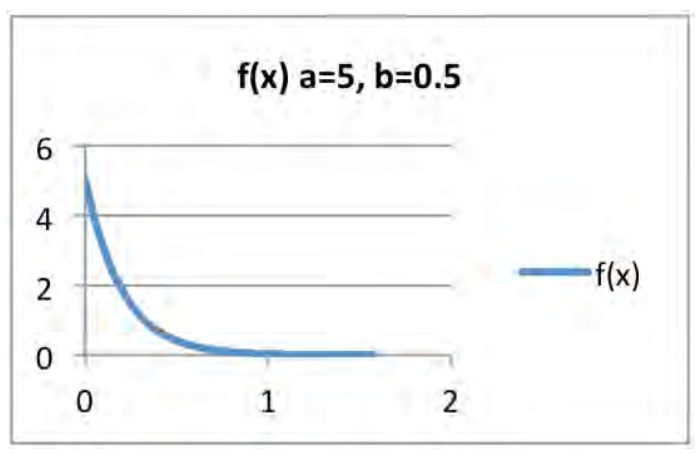

Figure 4. LFRD Density function when $a=5, b=0.5$ 


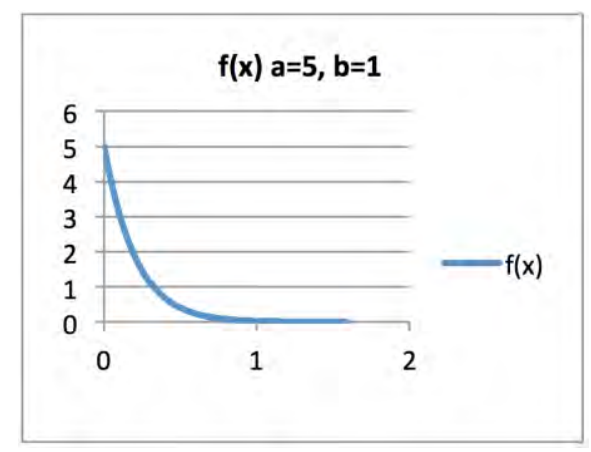

Figure 5. LFRD Density function when $a=5, b=1$

In Figures $1-5$, the combinations of $a$ and $b$ are bound by $a^{2}>b$, accordingly the mode is zero and the graphs are similar to that of exponential distribution. These characteristics of LFRD and its component distribution-exponential, motivated us to study the discriminatory aspect between LFRD and exponential through statistical test procedures. Such studies of discriminatory problems between probability models are made by Gupta, et al. (2002), Gupta and Kundu (2003a), Gupta and Kundu (2003b), Kundu and Gupta (2004a, 2004b), Kundu and Manglick (2004), Kundu, et al. (2005), Kundu and Manglick (2005), Kundu (2005), Kundu and Raqab (2007), Arabin and Kundu (2009), Arabin and Kundu (2010), Arabin and Kundu (2012a), Arabin and Kundu (2012b) and the references therein. The rest of the article is organised as follows. The methodology of the proposed LR type criterion for testing is described in the next section. The critical values of the test statistic are presented in following section. The aspects of power of the proposed test statistic are given in the final section, with a comparative study.

\section{LR Type Methodology}

Consider LFRD as a null population for example, $P_{0}$, the exponential model is regarded as an alternative population such as $P_{1}$. Let $x_{1}, x_{2}, \ldots, x_{n}$ be a given random sample of size $\mathrm{n}$. Let $L_{1}$ denote the value of the likelihood function at the sample $x_{1}, x_{2}, \ldots, x_{n}$ with reference to the population $P_{1} . L_{1}$ is obtained as follows. Considering $x_{1}, x_{2}, \ldots, x_{n}$ as a sample from $P_{1}$ with some method of point estimation using the $P_{1}$ as the mathematical model, substituting the values of the 


\section{KANTAM ET AL}

estimates so obtained and the sample observations $x_{1}, x_{2}, \ldots, x_{n}$ in $L_{1}$ results in a value of $L_{1}$ from the sample $x_{1}, x_{2}, \ldots, x_{n}$ with respect to $P_{1}$. Using the sample $x_{1}, x_{2}, \ldots, x_{n}$ with $P_{0}$ as the model one can get estimates of the parameters of $P_{0}$ thereby getting the value of the likelihood function in relation to $P_{0}$ at $x_{1}, x_{2}, \ldots, x_{n}$ the parameters of $P_{0}$ as estimated using $x_{1}, x_{2}, \ldots, x_{n} . L_{0}$ is thus the value of likelihood function substituting the same sample $x_{1}, x_{2}, \ldots, x_{n}$ and the estimates of $P_{0}$. Thus for the same sample $x_{1}, x_{2}, \ldots, x_{n}$, two values of likelihood function with respect to $P_{0}$ as well as $P_{1}$ were obtained.

Generally in likelihood ratio test procedure the MLEs of the parameters in $L_{1}$ and $L_{0}$ are substituted thereby getting the value of $L_{1} / L_{0}$ at a given samples $x_{1}, x_{2}, \ldots, x_{n}$ with the parameters of $P_{1}, P_{0}$ estimated by ML method using the respective models. Because likelihood is also joint probability of the sample $x_{1}, x_{2}, \ldots, x_{n}$, had the sample belonged to $P_{0}$ the ratio $L_{1} / L_{0}$ tends to be very small. If it is the other way - that is the sample is truly from $P_{1}$-then the ratio $L_{1} / L_{0}$ tends to be very large. Hence the ratio $L_{1} / L_{0}$ can be a criterion to test whether the sample $x_{1}, x_{2}, \ldots, x_{n}$ actually belongs to the population $P_{1}$ or $P_{0}$. If $L_{1} / L_{0}$ is very small it may be stated that the sample belongs to $P_{0}$. Thus the ratio $L_{1} / L_{0}$ decides the sample to have belonged to either $P_{1}$ or $P_{0}$. It is therefore necessary to get critical values for $L_{1} / L_{0}$ to decide whether a given sample belongs to $P_{1}$ or $P_{0}$. In turn this leads to the knowledge of percentiles of the sampling distribution of $L_{1} / L_{0}$. In the proposed method of testing LFRD vs. exponential, point estimates of the parameters were used in both null and alternative populations using any other point estimation instead of the classical ML method, because MLEs of LFRD parameters are not analytically available. Similar testing processes were adopted by other researchers (Gupta \& Kundu, 2003a; Kundu, et al., 2005). The proposed method is named the LR Type Criterion. In the discussion, the methods of point estimation that are considered are Least Squares estimators, Percentiles estimators, and Weighted Least Squares Estimators. The sampling distribution of $L_{1} / L_{0}$ is not mathematically tractable. The percentiles of $L_{1} / L_{0}$ were obtained through MonteCarlo simulation as described in the following section. For comparison purposes, the following parametric combinations were chosen. 


\section{LIKELIHOOD RATIO TYPE TEST FOR LINEAR FAILURE RATE}

Table 1. Parametric combinations chosen for the study.

\begin{tabular}{|c|c|c|c|c|c|}
\hline \multicolumn{2}{|c|}{ Least Squares Estimators } & \multicolumn{2}{|c|}{ Percentiles Estimators } & \multicolumn{2}{|c|}{$\begin{array}{c}\text { Weighted Least Squares } \\
\text { Estimators }\end{array}$} \\
\hline$a$ & $b$ & $a$ & $b$ & $a$ & $b$ \\
\hline 0.5 & 4.0 & 0.5 & 4.0 & 0.5 & 4.0 \\
\hline 2.5 & 0.5 & 2.5 & 0.5 & 2.5 & 0.5 \\
\hline 3.0 & 0.5 & 3.0 & 0.5 & 3.0 & 0.5 \\
\hline 3.5 & 1.0 & 3.5 & 1.0 & 3.5 & 1.0 \\
\hline 5.0 & 0.5 & 5.0 & 0.5 & 5.0 & 0.5 \\
\hline 5.0 & 1.0 & 5.0 & 1.0 & 5.0 & 1.0 \\
\hline
\end{tabular}

\section{LR Type Test Statistic - Critical Values}

A random sample of size $n$ is generated from LFRD $\left(P_{0}\right)$ with parameter combinations as specified in the Table 1. Using that sample the parameters of LFRD are estimated by least square method / percentile method / weighted least square method given method of estimation. The estimates so obtained are substituted in $P_{0}$ in the respective places of the parameters along with the sample observations used to get those estimates thus having an estimated value of $L_{0}$. Using the same sample, the parameters appearing in $P_{1}$ are estimated by a least square method / percentile method / weighted least square method in succession using the model $P_{1}$ method suitable for $P_{1}$. Here because $P_{1}$ is an exponential distribution the MLEs of parameters of $P_{1}$ were calculated using formulae and expressions suitable for $P_{1}$. The estimates of the parameters of $P_{1}$ so obtained are then substituted in $P_{1}$ along with the sample observations used to get the estimates. Thus estimated likelihood function $L_{1}$ are obtained by three separate methods. The ratio $L_{1} / L_{0}$ for different samples with the same parameter combinations as described in the previous section is calculated for each sample. This procedure was repeated 10,000 times for accuracy and precision. Among these 10,000 values, various specified cut off points (percentiles) would form the critical values of $L_{1} / L_{0}$ useful for testing. These are given below in the following Tables 2 and 3 , for only the parameters $(a=2.5, b=0.5),(a=3, b=0.5)$. Results of other parameter combinations are available from the authors. 
KANTAM ET AL

Table 2a: Percentiles of $L_{1} / L_{0}:: P_{0}$ : LFRD vs $P_{1}$ : EXP, Least Square Estimation, (a=2.5, $b=0.5$ )

\begin{tabular}{ccccc} 
& \multicolumn{5}{c}{ Least Square Estimation } \\
\cline { 2 - 5 } $\boldsymbol{n}$ & $\mathbf{5}$ & $\mathbf{1 0}$ & $\mathbf{1 5}$ & $\mathbf{2 0}$ \\
\hline 0.00100 & 0.05555 & 0.00866 & 0.00641 & 0.00495 \\
0.00135 & 0.05579 & 0.00980 & 0.00700 & 0.00551 \\
0.00270 & 0.05802 & 0.01389 & 0.01311 & 0.01005 \\
0.00500 & 0.06338 & 0.01944 & 0.01997 & 0.01839 \\
0.01000 & 0.07127 & 0.03196 & 0.03634 & 0.04059 \\
0.02500 & 0.09607 & 0.07852 & 0.09572 & 0.09172 \\
0.05000 & 0.15049 & 0.17091 & 0.17663 & 0.16909 \\
0.10000 & 0.27829 & 0.32933 & 0.33165 & 0.32362 \\
0.90000 & 1.45170 & 1.30077 & 1.31607 & 1.35776 \\
0.95000 & 2.36966 & 1.56559 & 1.55775 & 1.59782 \\
0.97500 & 4.97214 & 2.00069 & 1.86525 & 1.85212 \\
0.99000 & 20.67554 & 3.27230 & 2.50671 & 2.34857 \\
0.99500 & 89.41741 & 6.02098 & 3.90709 & 3.01258 \\
0.99730 & 206.88170 & 10.79545 & 5.50735 & 4.79198 \\
0.99865 & 938.89170 & 20.64189 & 19.63486 & 12.46792 \\
0.99000 & 1441.98200 & 40.78289 & 23.69878 & 36.68090 \\
\hline
\end{tabular}

Table 2b: Percentiles of $L_{1} / L_{0}:: P_{0}$ : LFRD vs $P_{1}$ : EXP, Weighted Least Square Estimation, $(a=2.5, b=0.5)$

Weighted Least Square Estimation

\begin{tabular}{ccccc}
$\boldsymbol{n}$ & $\mathbf{5}$ & $\mathbf{1 0}$ & $\mathbf{1 5}$ & $\mathbf{2 0}$ \\
\cline { 2 - 5 } 0.00100 & 0.05558 & 0.00865 & 0.00560 & 0.00541 \\
0.00135 & 0.05639 & 0.00958 & 0.00619 & 0.00693 \\
0.00270 & 0.06081 & 0.01370 & 0.01244 & 0.00971 \\
0.00500 & 0.06562 & 0.01851 & 0.01819 & 0.01855 \\
0.01000 & 0.07279 & 0.03215 & 0.03719 & 0.04195 \\
0.02500 & 0.09342 & 0.07797 & 0.09896 & 0.09637 \\
0.05000 & 0.14239 & 0.16794 & 0.17843 & 0.18065 \\
0.10000 & 0.26167 & 0.32321 & 0.33875 & 0.34647 \\
0.90000 & 1.42631 & 1.28926 & 1.36464 & 1.46510 \\
0.95000 & 2.39327 & 1.58677 & 1.65037 & 1.76804 \\
0.97500 & 5.02094 & 2.18780 & 2.14986 & 2.28297 \\
0.99000 & 19.63531 & 3.80238 & 4.02971 & 3.72403 \\
0.99500 & 88.76622 & 9.38806 & 9.31526 & 8.65864 \\
0.99730 & 222.91150 & 19.79771 & 28.90935 & 27.69206 \\
0.99865 & 825.53910 & 58.24844 & 314.41790 & 122.53770 \\
0.99000 & 1537.66000 & 125.22960 & 826.64140 & 388.92530 \\
\hline
\end{tabular}




\section{LIKELIHOOD RATIO TYPE TEST FOR LINEAR FAILURE RATE}

Table 2c: Percentiles of $L_{1} / L_{0}:: P_{0}$ : LFRD vs $P_{1}$ : EXP, Percentile Estimation, ( $a=2.5$, $b=0.5)$

\begin{tabular}{ccccc} 
& \multicolumn{4}{c}{ Percentile Estimation } \\
\cline { 2 - 5 } $\boldsymbol{n}$ & $\mathbf{5}$ & $\mathbf{1 0}$ & $\mathbf{1 5}$ & $\mathbf{2 0}$ \\
\hline 0.00100 & 0.07292 & 0.01208 & 0.00611 & 0.00468 \\
0.00135 & 0.07628 & 0.01361 & 0.00718 & 0.00559 \\
0.00270 & 0.08055 & 0.01710 & 0.00989 & 0.00979 \\
0.00500 & 0.08669 & 0.02131 & 0.01592 & 0.01877 \\
0.01000 & 0.09456 & 0.03308 & 0.03379 & 0.03679 \\
0.02500 & 0.12043 & 0.07695 & 0.08074 & 0.07452 \\
0.05000 & 0.16311 & 0.15190 & 0.15107 & 0.15069 \\
0.10000 & 0.24330 & 0.28240 & 0.28224 & 0.28175 \\
0.90000 & 2.08305 & 1.52860 & 1.46043 & 1.44967 \\
0.95000 & 4.89041 & 2.23917 & 1.97528 & 1.82456 \\
0.97500 & 14.79908 & 4.16817 & 3.02435 & 2.50522 \\
0.99000 & 123.33970 & 19.42763 & 7.95037 & 5.62689 \\
0.99500 & 748.87240 & 71.67762 & 31.90508 & 13.60665 \\
0.99730 & 2710.38500 & 246.98620 & 100.23880 & 55.98616 \\
0.99865 & 71595.25000 & 623.14900 & 454.89490 & 233.64480 \\
0.99000 & 190377.10000 & 897.07890 & 952.26130 & 833.10900 \\
\hline
\end{tabular}

Table 3a: Percentiles of $L_{1} / L_{0}:: P_{0}$ : LFRD vs $P_{1}$ : EXP, Least Square Estimation, (a=3.0, $b=0.5$ )

Least Square Estimation

\begin{tabular}{ccccc}
$\boldsymbol{n}$ & $\mathbf{5}$ & $\mathbf{1 0}$ & $\mathbf{1 5}$ & $\mathbf{2 0}$ \\
\cline { 2 - 5 } 0.00100 & 0.05603 & 0.01062 & 0.00596 & 0.00513 \\
0.00135 & 0.05691 & 0.01139 & 0.00662 & 0.00634 \\
0.00270 & 0.06038 & 0.01314 & 0.01177 & 0.01171 \\
0.00500 & 0.06443 & 0.02129 & 0.02265 & 0.02324 \\
0.01000 & 0.06995 & 0.03725 & 0.04223 & 0.04346 \\
0.02500 & 0.08877 & 0.09321 & 0.09890 & 0.09904 \\
0.05000 & 0.13725 & 0.17716 & 0.17834 & 0.18288 \\
0.10000 & 0.26580 & 0.33410 & 0.34319 & 0.33358 \\
0.90000 & 1.43639 & 1.33367 & 1.35457 & 1.37469 \\
0.95000 & 2.31841 & 1.62922 & 1.59357 & 1.61963 \\
0.97500 & 4.98869 & 2.14252 & 1.94302 & 1.93538 \\
0.99000 & 21.02987 & 4.00168 & 2.80106 & 2.50630 \\
0.99500 & 80.51004 & 8.20346 & 3.78306 & 3.15834 \\
0.99730 & 252.88440 & 20.03408 & 6.46744 & 3.97503 \\
0.99865 & 3116.18000 & 71.98767 & 11.33482 & 6.36183 \\
0.99000 & 59094.28000 & 179.53870 & 17.87834 & 7.78476 \\
\hline
\end{tabular}


KANTAM ET AL

Table 3b: Percentiles of $L_{1} / L_{0}:: P_{0}$ : LFRD vs $P_{1}$ : EXP, Weighted Least Square Estimation, $(a=3.0, b=0.5)$

\begin{tabular}{ccccc} 
& \multicolumn{4}{c}{ Weighted Least Square Estimation } \\
\cline { 2 - 5 } $\boldsymbol{n}$ & $\mathbf{5}$ & $\mathbf{1 0}$ & $\mathbf{1 5}$ & $\mathbf{2 0}$ \\
\hline 0.00100 & 0.05647 & 0.01066 & 0.00576 & 0.00664 \\
0.00135 & 0.05761 & 0.01104 & 0.00669 & 0.00795 \\
0.00270 & 0.06264 & 0.01395 & 0.01140 & 0.01456 \\
0.00500 & 0.06704 & 0.02150 & 0.02225 & 0.02212 \\
0.01000 & 0.07299 & 0.03735 & 0.04243 & 0.04817 \\
0.02500 & 0.08761 & 0.08531 & 0.09748 & 0.10723 \\
0.05000 & 0.12932 & 0.17186 & 0.18351 & 0.19496 \\
0.10000 & 0.25456 & 0.32422 & 0.34730 & 0.36366 \\
0.90000 & 1.42080 & 1.31768 & 1.38883 & 1.49770 \\
0.95000 & 2.32665 & 1.65014 & 1.67716 & 1.86482 \\
0.97500 & 4.88276 & 2.28681 & 2.14252 & 2.36452 \\
0.99000 & 20.92875 & 4.86321 & 3.67318 & 3.89300 \\
0.99500 & 72.31535 & 11.28078 & 6.79731 & 6.48627 \\
0.99730 & 281.68090 & 32.09840 & 21.00146 & 24.05159 \\
0.99865 & 2668.58100 & 204.66170 & 82.91345 & 187.71560 \\
0.99000 & 60999.62000 & 313.55800 & 123.97500 & 744.18340 \\
\hline
\end{tabular}

Table 3c: Percentiles of $L_{1} / L_{0}:: P_{0}$ : LFRD vs $P_{1}$ : EXP, Percentile Estimation, ( $a=3.0$, $b=0.5$ )

\begin{tabular}{ccccc}
$\boldsymbol{n}$ & \multicolumn{5}{c}{ Percentile Estimation } \\
\cline { 2 - 5 } & $\mathbf{5}$ & $\mathbf{1 0}$ & $\mathbf{1 5}$ & $\mathbf{2 0}$ \\
\hline 0.00100 & 0.07245 & 0.01317 & 0.00645 & 0.00460 \\
0.00135 & 0.07337 & 0.01435 & 0.00712 & 0.00557 \\
0.00270 & 0.08110 & 0.01997 & 0.01176 & 0.01181 \\
0.00500 & 0.08790 & 0.02728 & 0.01912 & 0.02171 \\
0.01000 & 0.09718 & 0.04102 & 0.03919 & 0.03662 \\
0.02500 & 0.11891 & 0.08040 & 0.08506 & 0.08822 \\
0.05000 & 0.16062 & 0.15065 & 0.15994 & 0.16028 \\
0.10000 & 0.25323 & 0.28654 & 0.30645 & 0.29008 \\
0.90000 & 2.06479 & 1.54506 & 1.49559 & 1.46275 \\
0.95000 & 5.04268 & 2.27774 & 1.96774 & 1.85958 \\
0.97500 & 14.98131 & 4.37480 & 3.03259 & 2.57786 \\
0.99000 & 95.64787 & 17.19165 & 7.48988 & 4.85088 \\
0.99500 & 765.44120 & 76.76962 & 19.14950 & 14.15270 \\
0.99730 & 4913.02900 & 229.08730 & 59.52394 & 70.34382 \\
0.99865 & 343286.90000 & 526.59070 & 325.89340 & 280.56010 \\
0.99000 & 2031568.00000 & 1125.17300 & 478.65110 & 711.98170 \\
\hline
\end{tabular}




\section{LIKELIHOOD RATIO TYPE TEST FOR LINEAR FAILURE RATE}

\section{LR Type Test Statistic - Power}

The LR type statistic suggested would be meaningful only if it is able to distinguish between the null and alternative populations. As is generally considered, the level of significance was fixed at 0.05 . The critical value of $L_{1} / L_{0}$ corresponding to the level of significance 0.05 is (corresponding to the percentile at 0.95 ) identified from the relevant portion of Tables 2 and 3.

10,000 random samples of size each $n=5$ (5) 20, from the alternative population (exponential) are generated. The MLE (reciprocal of sample mean) of the parameter of the alternative population, the individual sample values are substituted in $L_{1}$ to get the value of $L_{1}$. Using the same sample the value of $L_{0}$ as described in the previous section is also computed in order to get 10,000 values of $L_{1} / L_{0}$ for a given sample size, for a given parametric combination and for a given method of point estimation applied to the parameters of $P_{0}$. The proportion of values of $L_{1} / L_{0}$ that exceeded the critical value $\left(c_{0}\right)$ out of 10,000 is computed and is considered as the power of the test statistic at level of significance 0.05 .

Table 4. Powers of LR Test Criterion at $\alpha=0.05$ Parameter Estimates Using P.E., L.S.E., W.L.S.E. Methods

\begin{tabular}{|c|c|c|c|c|c|c|c|c|c|c|c|c|}
\hline \multirow[b]{3}{*}{$\begin{array}{l}\text { Parameter } \\
\text { Combinations }\end{array}$} & \multicolumn{12}{|c|}{ Estimation Method } \\
\hline & \multicolumn{4}{|c|}{ Percentile } & \multicolumn{4}{|c|}{ Least Squares } & \multicolumn{4}{|c|}{ Weighted Least Squares } \\
\hline & $n=5$ & $n=10$ & $n=15$ & $n=20$ & $n=5$ & $n=10$ & $n=15$ & $n=20$ & $n=5$ & $n=10$ & $n=15$ & $5 \quad n=20$ \\
\hline$a=2.5, b=0.5$ & 0.0539 & 0.0601 & 0.0606 & 0.0729 & 0.0598 & 0.0692 & 0.0735 & 0.0737 & 0.0587 & 0.0672 & 0.0646 & 60.0697 \\
\hline$a=3, b=0.5$ & 0.0516 & 0.0585 & 0.0608 & 0.0704 & 0.0612 & 0.0619 & 0.0676 & 0.0700 & 0.0608 & 0.0607 & 0.0609 & 90.0599 \\
\hline$a=3.5, b=1$ & 0.0534 & 0.0586 & 0.0613 & 0.0726 & 0.0632 & 0.0714 & 0.0740 & 0.0786 & 0.0621 & 0.0668 & 0.0806 & 60.0678 \\
\hline$a=5, b=0.5$ & 0.0505 & 0.0500 & 0.0533 & 0.0608 & 0.0581 & 0.0570 & 0.0592 & 0.0599 & 0.0589 & 0.0559 & 0.0525 & 50.0543 \\
\hline$a=5, b=1$ & 0.0505 & 0.0540 & 0.0549 & 0.0606 & 0.0920 & 0.0639 & 0.0645 & 0.0619 & 0.0571 & 0.0613 & 0.0534 & 40.0589 \\
\hline$a=0.5, b=4$ & 0.0517 & 0.1126 & 0.3692 & 0.6813 & 0.1987 & 0.4105 & 0.6137 & 0.7472 & 0.2018 & 0.0613 & 0.5280 & 00.6024 \\
\hline
\end{tabular}

A large value of the power shows that the test statistic is able to distinguish between the null and alternative populations. A small value of the power would show the indistinguishability between $P_{1}$ and $P_{0}$ as decided by LR type test statistic. The powers so obtained are given in Table 4, treated separately for each method of estimation at a specified level of significance 0.05 .

The tabulated power values are very poor touching a maximum of 0.092 at $n=5, a=5, b=1$. These recorded powers show that the LR type test statistic is not able to discriminate between LFRD and exponential at all the values of $n$ and the 


\section{KANTAM ET AL}

respective parametric combinations across the methods of estimation, except the last row of each table. It shows that exponential distribution can be used as an alternative for LFRD without much loss whereas the last row of each table shows that LFRD and exponential stand apart from each other for $a=0.5, b=4$. It is therefore concluded that the simple and powerful inferential tools available for exponential may be used for LFRD also. The discrimination between LFRD and exponential is clear as evident from the last row of each table.

\section{References}

Arabin K. D., \& Kundu, D. (2009). Discriminating among the log-normal, Weibull and generalized exponential distributions. IEEE Transactions on Reliability, 58(3): 416-424.

Arabin K. D., \& Kundu, D. (2010). Discriminating between the log-normal and log-logistic distributions. Communications in Statistics - Theory and Methods, 39: 280-292.

Arabin K. D., \& Kundu, D. (2012a). Discriminating Between the Weibull and Log-Normal Distributions for Type-II Censored Data. Statistics, 46(2): 197214.

Arabin K. D., \& Kundu, D. (2012b). Discriminating Between Bivariate Generalized Exponential and Bivariate Weibull Distributions. Chilean Journal of Statistics, 3(1): 93-110.

Bain, L. J. (1974). Analysis for the Linear Failure-Rate Life-Testing Distribution. Technometrics, 16(4): 551-559.

Gupta, R. D., \& Kundu, D. (2003a). Discriminating Between the Weibull and the GE Distributions. Computational Statistics and Data Analysis, 43: 179196.

Gupta, R. D., \& Kundu, D. (2003b). Closeness of Gamma and Generalized Exponential Distributions. Communications in Statistics - Theory and Methods, 32(4): 705-721.

Gupta, R. D., Kundu, D., \& Manglick, A. (2002). Probability of Correct Selection of Gamma Versus GE or Weibull vs. GE Models Based on Likelihood Ratio Test. In Y. P. Chaubey, Ed. Recent Advances in Statistical Methods (pp. 147-156). London: World Scientific Publishing Company Inc.

Kundu, D. (2005). Discriminating between the Normal and Laplace Distributions. In N. Balakrishnan, H. N. Nagaraja and N. Kannan (Eds.). 


\section{LIKELIHOOD RATIO TYPE TEST FOR LINEAR FAILURE RATE}

Advances in Ranking and Selection, Multiple Comparisons, and Reliability (pp. 65-78). Boston: Birkhauser.

Kundu, D., \& Gupta, R. D. (2004a). Discriminating Between the Gamma and Generalized Exponential Distributions. Journal of Statistical Computation and Simulation, 74(2): 107-121.

Kundu, D., \& Gupta, R. D. (2004b). Discriminating Between the Weibull and Log-Normal Distributions. Naval Research Logistics, 51(6): 893-905.

Kundu, D., Gupta, R. D., \& Manglick, A. (2005). Discriminating Between the Log-Normal and the Generalized Exponential Distributions. Journal of Statistical Planning and Inference, 127: 213-227.

Kundu D., \& Manglick, A., (2004). Discriminating between the Weibull and Log-Normal Distributions. Naval Research Logistics, 51(6): 893-905.

Kundu, D., \& Manglick, A. (2005). Discriminating Between the gamma and log-normal Distributions. Journal of Applied Statistical Sciences, 14(1-2): 175187.

Kundu, D., \& Raqab, M. Z. (2005). Generalized Rayleigh Distribution: Different Methods of Estimation. Computational Statistics and Data Analysis, 49: 187-200.

Kundu, D., \& Raqab, M. Z. (2007). Discriminating Between the LogNormal and Generalized Rayleigh Distributions. Statistics, 41(6): 505-515.

Sen, A. (2005). Linear failure rate distribution. In Kotz, Balakrishnan, Read and Vidakovic, Eds. Encyclopedia of Statistical Sciences, (Vol. 6), pp. 4212-4217. 